Romero-Rodríguez, S., Figuera-Gazo, P., Freixa-Niella, M. y Llanes-Ordóñez, J. (2019). Adaptabilidad de la carrera en estudiantes universitarios: un estudio a través de entrevistas autobiográficas. Revista de Investigación Educativa, 37(2), 379-394.

DOI: http://dx.doi.org/10.6018/rie.37.2.322441

\title{
Adaptabilidad de la Carrera en estudiantes universitarios: Un estudio a través de entrevistas autobiográficas'
}

\section{Career adaptability among University students:A study through autobiographical interviews}

\author{
Soledad Romero-Rodríguez*, Pilar Figuera-Gazo**, Montserrat Freixa-Niella**** \\ y Juan Llanes-Ordóñez ${ }^{* * * * *}$ \\ *Departamento de Métodos de Investigación y Diagnóstico en Educación. Facultad de Ciencias de la Educación. \\ Universidad de Sevilla (Spain) \\ **,***,***** Departamento de Métodos de Investigación y Diagnóstico en Educación. Facultad de Educación. \\ Universidad de Barcelona (España)
}

\begin{abstract}
Resumen
En este trabajo se presenta un estudio de casos cualitativo en el que se analizan las trayectorias de estudiantes universitarios a través de entrevistas autobiográficas. La finalidad ha sido comprender los recursos y estrategias de adaptabilidad que utiliza el estudiantado y su evolución. Las entrevistas se realizaron a 17 personas graduadas en Pedagogía y Administración y Dirección de Empresas. El análisis de las entrevistas se realizó a través de la identificación de las cuatro dimensiones propuestas por Savickas y Porfeli (2012) para la evaluación de la adaptabilidad: Curiosidad, Control, Confianza e Implicación. Los resultados apoyan la aportación del uso de metodologías narrativas en el estudio de las transiciones y la adaptabilidad de la carrera. La discusión se centra en la diferente presencia de estas dimensiones en las trayectorias del estudiantado y las carencias que justifican la necesidad de realizar intervenciones orientadoras narrativas para potenciar el desarrollo de la carrera en la Universidad.

Palabras clave: desarrollo profesional, estudiantes universitarios, transición, investigación cualitativa, orientación profesional, orientación.

1 Proyecto "Trayectorias de abandono, persistencia y graduación en Ciencias Sociales: validación de un modelo predictivo" financiado en el marco del Plan Nacional I+D (EDU2012-31568).

Correspondencia: Soledad Romero-Rodríguez, sromero@us.es, Universidad de Sevilla, Facultad de Ciencias de la Educación, Departamento de Métodos de Investigación y Diagnóstico en Educación, C/ Pirotecnia, s/n, 41013, Sevilla
\end{abstract}




\begin{abstract}
This research reports a qualitative case study in which university students' career paths are analyzed through autobiographical interviews. Seventeen graduates of pedagogic sciences and business administration and management were selected. Our aim was to analyze the students' adaptabilities and resources to cope with and adapt to changes during their transition processes. A deductive analysis of career adaptability was conducted. The dimensions proposed by Savickas and Porfely (2012) were carried out in order to assess adaptability: Curiosity, Control, Confidence and Concern. The results support the contribution of qualitative and narrative research in the study of transitions and career adaptability. The discussion focuses on the different presence of the four dimensions in each case and justifies the need of developing narrative career interventions at university.
\end{abstract}

Keywords: career development; university Students; transition; qualitative research; career counselling; guidance.

\title{
Introducción
}

Los procesos de transición durante la trayectoria universitaria, desde que el estudiantado toma la decisión de realizar los estudios hasta que se culminan, son una oportunidad para la construcción de la carrera. Sin embargo, aún son escasas las investigaciones que se han planteado como objetivo aportar información relevante para que el acompañamiento que requiere el estudiantado en este proceso sea efectivo (Álvarez, López \& Pérez-Jorge, 2015; Martínez-Clares, Pérez-Cusó \& Martínez-Juárez, 2014).

Estudios previos apuntan a la importancia de realizar un acercamiento ecológico y sistémico a los procesos de transición universitaria, tratando de comprender cómo construye cada estudiante la narración de su propia trayectoria en el presente y cómo se proyecta profesionalmente (Romero-Rodríguez, Figuera-Gazo \& Freixa-Niella, 2017).

La narración de la propia historia vital permite dar sentido y coherencia a todo lo que se vive y experimenta (Bernal \& Cárdenas, 2009), lo que cobra especial importancia en un momento en el que las transiciones están marcadas por la incertidumbre y los constantes y acelerados cambios tecnológicos, culturales y sociales (González Lorente \& Martínez Clares, 2016). La narrativa funciona como un puente a través del cual se van construyendo identidades provisionales en un proceso que cuenta con una dimensión social y en las que pueden coexistir diferentes relatos a un mismo tiempo (lbarra \& Barbulescu, 2010).

La Teoría de la Construcción de la Carrera (CCT) aporta una perspectiva constructivista y narrativa del desarrollo y la orientación para la carrera. En el marco de esta teoría, la adaptabilidad de la carrera es un constructo psicosocial de carácter multidimensional que incluye la disponibilidad y los recursos necesarios para afrontar con éxito las tareas de desarrollo de la carrera, responder de manera positiva a las transiciones que se dan a lo largo de la vida y mejorar el bienestar personal. Cada nueva experiencia forma una pequeña historia en la narrativa de la vida de una persona. La forma en que se narra esta historia revela cómo la persona se va adaptando a las situaciones que debe afrontar cuando toma decisiones (Del Corso y Rehfuss, 2011). Las estrategias y los recursos personales (adapt-abilities) que les ayudan a afrontar los cambios (previstos o 
no) y a adaptarse a cada nueva situación, se organizan en cuatro dimensiones. Cada una de ellas hace referencia a una serie de capacidades de autorregulación (Savickas \& Porfeli, 2012). La implicación muestra el compromiso de la persona con la toma de decisiones de futuro y la planificación de las acciones para llevarlas a cabo; el control incluye la conciencia de la responsabilidad personal en la toma de decisiones y su ejecución; la curiosidad lleva a la persona a explorar la mayor posibilidad de alternativas de futuro y de las acciones a emprender para alcanzarlas y la confianza aporta a la persona el sentimiento de autoeficacia para alcanzar sus objetivos y superar obstáculos. Para medir la adaptabilidad de la carrera, se diseñó la Career Adapt-Abilities Scale (Savickas \& Porfeli, 2012), adaptada en más de 8 lenguas, en numerosos países y para poblaciones diversas (Johnston, 2018; Savickas \& Guichard, 2016).

La globalización, las crisis socioeconómicas, la permeabilidad de las fronteras de las carreras, la inestabilidad en el empleo, en definitiva, las características de la sociedad líquida postmoderna, hacen necesario que la persona desarrolle las competencias requeridas para construir su proyecto profesional, así como la capacidad para gestionar su carrera en contextos de incertidumbre (Nota \& Rossier, 2015). La CCT propone una secuencia de adaptación en la que la disponibilidad adaptativa (adaptivity) influye en los recursos y capacidades para la adaptación (adaptability), a la vez que ésta incide en las respuestas adaptativas (adapting) y en los resultados de la adaptación (adaptation). Las relaciones entre estas cuatro etapas han sido analizadas en diferentes investigaciones y ayudan a comprender la adaptabilidad. Perera y McIlveen (2017) muestran la diferencia que existe en la relación entre estas fases en función de los perfiles personales de predisposición al cambio ("preparados para el cambio", "rígidos" y "ordinarios"). Hamtiaux, Houssemand y Vrignaud (2013) concluyen sobre la importancia de la situación contextual como moderador de la incidencia de los recursos de adaptabilidad en las respuestas adaptativas. Rudolph, Lavigne y Zacher (2017) desarrollan un meta-análisis en que se manifiesta que los recursos de la adaptabilidad de la carrera facilitan respuestas adaptativas (planificación, exploración y toma de decisiones) frente a situaciones cambiantes. Asimismo, influyen positivamente en la empleabilidad, la disminución del estrés laboral, el compromiso, el emprendimiento y el bienestar y la satisfacción personal en diferentes roles vitales, no solo laborales.

Rudolph, Lavigne, Katz y Sacher (2017) se han centrado en la incidencia específica y diferencial de cada una de las cuatro dimensiones de la adaptabilidad de la carrera en los resultados de adaptación. Concluyen que el grado en que incide cada una de las dimensiones en las diferentes respuestas adaptativas varía en tamaño y magnitud de manera significativa, mientras que en otras respuestas la incidencia es interactiva y multidimensional. En una misma persona, la forma e intensidad de las manifestaciones de cada una de las dimensiones de la adaptabilidad de la carrera puede fluctuar en un mínimo periodo de tiempo (Zacher, 2016).

Johnston (2018) ha analizado los resultados hallados por 116 investigaciones publicadas posteriormente a septiembre de 2015, en relación con los recursos de adaptabilidad y las respuestas de adaptación. Concluye que ambos elementos contribuyen a que las transiciones y el funcionamiento personal sean positivos, tanto en adolescentes como en personas adultas. Aporta, además, un exhaustivo análisis de factores que correlacionan o predicen la adaptabilidad y los resultados a los que lleva ésta. 
El contexto universitario ha sido uno de los preferidos en los estudios que se han realizado sobre la adaptabilidad de la carrera. Se han analizado la relación que tiene con factores como la vocación (Douglass \& Duffy, 2015); papel de la familia y sentimiento de autoeficacia (Guan, Capezio, Restubog, Read, Lajom \& Li, 2016); personalidad proactiva (Jiang, 2017); experiencias extracurriculares y transición al trabajo (Monteiro \& Da Silva, 2015); compromiso con la carrera y personalidad (Nilforooshan \& Salimi, 2016); inteligencia cultural y disponibilidad para la movilidad (Presbitero \& Quita, 2017) o intereses profesionales (Śverko \& Babarović, 2016). También en el contexto universitario se ha realizado la evaluación de intervenciones que se plantean como objetivo el desarrollo de la adaptabilidad de la carrera (Amado et al., 2017). A nivel nacional, destacan los trabajos de Merino-Tejedor, Hontangas y Boada-Grau (2016) y Merino-Tejedor, Hontangas y Petrides (2017).

Si bien son escasas las investigaciones de corte cualitativo, su revisión apunta a que la metodología narrativa es una línea interesante para comprender el proceso de construcción de la carrera y, específicamente, la adaptabilidad. Así lo muestran la revisión realizada por Bimrose y Hearne (2012) o los trabajos desarrollados sobre trayectorias de mujeres adultas (Bimrose et al. 2014; McMahon, Watson \& Bimrose, 2012; Whiston, Feldwisch, Evans, Blackman \& Gilman, 2015), sobre las transiciones a mediana edad (Brown, Bimrose, Barnes \& Hughes, 2012) o en la adultez emergente (Shulman, Vasalampi, Barr, Livne, Nurmi \& Pratt, 2014). También queda patente la aportación de este enfoque metodológico en el estudio de la construcción de las identidades estudiantiles y profesionales (McAdams \& Guo, 2014) o en el análisis de las transiciones desde un enfoque de la orientación a lo largo de la vida (Barabasch \& Merrill, 2014). Trabajos como el de Santos y Cardenal (2012) aportan bastantes argumentos sobre la legitimidad de la investigación autobiográfica en el actual escenario universitario.

En este trabajo se pretende:

1) Comprender los recursos y estrategias de adaptabilidad que utilizan los estudiantes universitarios;

2) Analizar la evolución de estos recursos a lo largo de su trayectoria académica.

\section{Método}

\section{Diseño}

Se ha desarrollado un estudio cualitativo de casos múltiple (Yin, 1984) utilizando como procedimiento de recogida de información la entrevista autobiográfica. Los relatos autobiográficos de recién graduados universitarios permiten la identificación de las estrategias y recursos que utiliza el estudiantado para adaptarse a los cambios (previstos o no) a los que debe hacer frente durante su trayectoria (adaptabilidad de la carrera).

\section{Participantes}

Se realizó un muestreo teórico (Glaser \& Strauss, 2009) atendiendo a la diversidad de puntos de vista de los informantes. Se seleccionaron 17 casos, atendiendo a los criterios: titulación (8 Pedagogía y 9 Administración y Dirección de Empresas-ADE); 
sexo (10 mujeres y 7 hombres); vía de acceso (3 CFGS, 4 abandono de primera titulación; 10 Bachillerato). Los participantes debían estar recién graduados (8 trayectorias de éxito) o bien haber superado el tiempo teórico de realización de los estudios (9 trayectorias de retraso).

\section{Instrumento: La entrevista autobiográfica}

El protocolo de la entrevista (Freixa \& Dorio, 2015) incluyó: datos biográficos; capítulos de su vida (antecedentes formativos, elección de estudios, transición a la Universidad, construcción de su identidad, cambios en la situación familiar, económica, curricular, institucional, clima de aula, profesorado) y narración de un capítulo de futuro (expectativas laborales y desarrollo profesional).

\section{Procedimiento de recogida y análisis de datos}

Las entrevistas se desarrollaron de manera presencial, previo acuerdo entre investigadores y entrevistados. Las entrevistas fueron grabadas, transcritas y contrastadas con los informantes.

El análisis se desarrolló a través del método de comparaciones constantes (Glaser \& Strauss, 2009), realizando una primera codificación y categorización independiente por dos investigadores y posterior revisión de la codificación por otras dos investigadoras. De este análisis se derivó un sistema de categorías que incluía factores que intervienen de manera sistémica en la construcción de la carrera, entre ellos, la adaptabilidad.

En una segunda codificación se atendió a las dimensiones de la adaptabilidad de la carrera según el modelo de Savickas (Savickas y Porfeli, 2012), identificando las categorías recogidas en la tabla 1.

Para asegurar la calidad del proceso se ha atendido esencialmente a la reflexividad. Se realizó una triangulación entre investigadores, en el análisis de datos, buscando la coherencia entre los resultados y las narraciones del estudiantado (Sandín, 2000, 2010).

Tabla 1

Sistema de categorías

\begin{tabular}{lll}
\hline Dimensión & \multicolumn{1}{c}{ Descriptores } & \multicolumn{1}{c}{ Categorías } \\
\hline Curiosidad & Exploración de alternativas & Importancia de la exploración \\
& & Estrategias de exploración \\
& & Utilización de espacios formativos \\
& Evolución de la curiosidad \\
Control & $\begin{array}{l}\text { Responsabilidad en la toma de } \\
\text { decisiones }\end{array}$ & $\begin{array}{l}\text { Persistencia } \\
\text { Conciencia de la responsabilidad }\end{array}$ \\
& & Evolución del control \\
\hline
\end{tabular}




\begin{tabular}{lll}
\hline Dimensión & \multicolumn{1}{c}{ Descriptores } & \multicolumn{1}{c}{ Categorías } \\
\hline Confianza & Sentimiento de autoeficacia & Eficiencia \\
& & Asunción del error \\
& Orgullo \\
& & Evolución de la confianza \\
Implicación & Compromiso con la toma de & Anticipación \\
& decisiones & Compromiso con el proyecto personal \\
& & Evolución de la implicación \\
\hline
\end{tabular}

\section{Aspectos éticos de la investigación}

Se protegieron los datos de las personas entrevistadas, utilizando nombres ficticios. Manifestaron el consentimiento informado antes de la realización de las entrevistas.

\section{Resultados}

Presentamos a continuación los resultados en función de los datos obtenidos para cada una de las dimensiones.

\section{Curiosidad}

En algunos estudiantes se observa una actitud constante de exploración, movidos por una necesidad de indagar sobre alternativas de estudio. Gisela parte de un movimiento interno de búsqueda ("...no tenía un estado de tranquilidad, de estar bien conmigo misma y comienzo un proceso más interno..."). Irina se mueve por una necesidad de vivir nuevas experiencias, a pesar de los miedos ("Me apetecía cambiar de aires. ...y sentía miedo del desconocimiento,").

Sin embargo, el comportamiento exploratorio no parece estar muy presente en algunas de las narraciones. Ivana llega a Pedagogía sin saber en qué consiste ("...No sabía bien qué era la pedagogía, pero me apunté"). Jano tenía claro que su objetivo era ir a la Universidad, pero no se preocupa por recopilar información sobre ADE y se decepciona ("La carrera no la he encontrado tal como me imaginaba"). En algunos casos, continúan basando sus decisiones a lo largo de su trayectoria en suposiciones y creencias que no contrastan a través de la exploración (Paloma: "Inocente de mí, pensé que el TFG era igual en todas las universidades..."). Yadira reconoce su dificultad para explorar y resolver sus dudas ("Y si no entiendo algo me da mucha vergüenza preguntarlo; error mío..."). El contacto con la realidad les lleva a la necesidad de observar, indagar, reflexionar, para poder adaptarse a la nueva situación (Daniel: "He pegado un progreso que me ha puesto los pies más en la tierra...con mucha más reflexión"; Mario: "Le diría que trabajara, que hiciera voluntariados y que contrastara todo lo que está aprendiendo con su proyecto de trabajo futuro"). Este proceso de exploración que parte de necesidades externas es vivido como 
un "buscarse la vida" (Ricardo) utilizando diferentes medios como Internet, la web de la Universidad o el plan de estudios (Servando).

Destacan las prácticas como espacio formativo que abre la posibilidad de motivar un proceso interno de observación, reflexión y orientación hacia el futuro, ayudando a desarrollar conciencia profesional (Neus: “...Una experiencia en constante construcción y apropiación de una realidad educativa"; Jano: "...Te permite un poco levantar la vista y decir: 'ostras, todo lo que estoy haciendo te lleva a un sitio'... tiene un final, la vida profesional").

Expresan la conciencia de que el proceso de exploración debe intensificarse al finalizar los estudios (Paloma: "Cuando acabas la carrera lo tienes todo y sabes de todo, pero no sabes de nada").

\section{Control}

La persistencia se manifiesta en la idea de que lo que se comienza hay que terminarlo, lo que, a la vez, les dificulta el replantearse su trayectoria cuando ésta no es coherente con sus intereses (Ivana: "Soy constante porque todo lo que comienzo lo acabo... pienso que podría ser más feliz siendo peluquera"). Daniel expresa que se mantiene en los estudios de Pedagogía a pesar de que le preocupa la falta de prestigio de esta titulación, porque "es de los que termina lo que empieza". Montse reconoce que realizó una elección equivocada, basada en la creencia en mitos, pero no se permite reconducir su trayectoria porque sería un "fracaso" y la ruptura de su imagen de "buena estudiante".

Se plantean metas como acabar la carrera en un número de años determinados, lo que no siempre pueden cumplir, con la consecuente frustración (Paloma: "Yo siempre he sido una persona persistente y metódica, entonces yo me propuse terminar la carrera en 4 años" y siente frustración cuando, debido al fallecimiento de su abuelo, tiene problemas con algunas asignaturas). En otros casos, el resultado es satisfactorio, aunque el proceso fue difícil y exento de planificación (Irina: "lo mal que lo pasé me valió para lo que conseguí... Es que tampoco quise dejar nada"). La constancia de Yago se manifiesta en su insistencia en hablar una lengua que no domina, preguntar en clase, a pesar de su conciencia del error.

Los participantes muestran la asunción de la responsabilidad de sus propias acciones y cómo van adaptando su actuación pasando por un proceso reflexivo a partir de las exigencias y requerimientos (externos) con los que se van encontrando (Yago: “... Vi cómo ser eficiente... Aprendí a estudiar con pocas horas, pero muy intensamente... Eso es espabilar totalmente porque ya me sentía muy bien en la 'uni'"; Ricardo: “...en vez de sentarme tan atrás me senté en primera fila yo solo. También estudiaba cada día un poco y luego a la época de finales me iba a la biblioteca y hacía el día allí"). A veces, intentan emprender acciones de mejora, pero lo hacen sin contar con la información suficiente (Paz: "Y cuando me cojo una asignatura de cuarto, solo está disponible el grupo en inglés. Entonces me voy a las academias y me pago un curso de finanzas... voy al examen y está en inglés. No me sirvió de nada").

Neus reconoce haber ido madurando en cuanto a tomar las riendas de su carrera: "El proceso que he vivido aquí lo describo como metamorfosis, no sólo he cambiado por fuera, sino por dentro: la manera de reflexionar, de trabajar, de relacionarme, de autonomía, de res- 
ponsabilidad, de tomar decisiones, de saber gestionarme, de interactuar, de buscar, de investigar y de sembrar mi propio camino me ha hecho madurar".

Esta evolución no se da en todos los casos. Nubia atribuye sus éxitos y fracasos a causas externas (su tutora, calificación de la nota de acceso, decepción porque la Universidad no se parece a la imagen de las películas...) y reconoce el peso que tienen en sus decisiones las expectativas de los demás: "tengo miedo al fracaso, pero por todas las expectativas que tienen los de fuera".

\section{Confianza}

El sentimiento de autoeficacia se ve mediatizado por la autoexigencia, que lleva a algunos de nuestros entrevistados a desconfiar de sus propias potencialidades y no reconocer sus errores, perdiendo eficacia en el aprovechamiento de oportunidades. En este proceso tiene un lugar muy importante la respuesta a las expectativas de los otros. Neus, aun reconociendo el dominio que tiene de ciertas competencias, tiene miedo a equivocarse y defraudar ("Sé que tengo también recursos y estrategias, pero es el miedo a defraudar a la persona que me haya contratado"). Paloma narra su inseguridad ("... Soy bastante insegura de mí misma..., me gusta tenerlo todo muy controlado y mi carácter me impide dar el paso ahora mismo").

Servando narra su sentimiento de frustración cuando observa que su método de estudio no le es válido para responder a las exigencias académicas y culpabiliza a las circunstancias ("Vi que mi modus operandi no funcionaba, me agobié... yo culpé al contexto... lo culpé por excusarme y no querer sentirme culpable").

El peso de la autoexigencia aparece también en Candela ("Me exijo mucho y a veces no me tendría que castigar tanto"). Jano asume la necesidad de relativizar la exigencia ("Que no te vaya tan bien como tu esperes no significa que no seas bueno para esto. Hay que relativizar"), después de haber vivido la experiencia de culpabilizarse por la sensación de fracaso.

La asunción del error actúa como acicate en la trayectoria de Biel. Tras abandonar los estudios en Bachillerato decide reingresar y adopta como reto el demostrar que "soy más de lo que aparento" y toma el voluntariado y la pedagogía como un proceso de contante búsqueda de experiencias de aprendizaje ("...Experiencias de vida, como la dinámica del casal que es de participación, de autogestión, de implicación propia, en la universidad cuando entro busco este tipo de lugares de participación y me encuentro con los representantes de los estudiantes, implicándose en junta, el Consell d'Estudis").

Los entrevistados muestran cómo la confianza en sus posibilidades, expresadas como "yo puedo", nace en algunos momentos de la comparación con los otros. Paz cuenta: “... Empiezo tercero y me matriculo de todas, yo trabajando, pero diciéndome a mí misma "yo puedo"; para mí era importante no quedarme atrás" lo que la lleva a situaciones que ponen en riesgo la continuación de sus estudios. Otras veces el "yo puedo" surge del convencimiento interno de que, a pesar de la desmotivación hay que acabar lo que se ha hecho (Ricardo: "Búscate una motivación por tu cuenta y acaba...haz lo que puedas para tirar").

El orgullo del trabajo bien realizado aparece como un elemento de confianza en sí mismo. (Irina: “...Es que por mis narices que lo saco...era el orgullo de decir que lo voy a hacer porque lo quiero hacer"). Servando expresa lo que le mueve a mantenerse en la titulación: "Orgullo, de poder decirles que me lo he sacado". Daniel intenta dar una imagen 
de seguridad, pero en su discurso aparecen sus miedos y frustraciones ("Soy positivo, y cojo las cosas con ganas, pero si esa cosa no está clara y no la veo muy bien tiendo incluso a tener miedo al fracaso"). Solo cuando percibe su trayectoria como positiva, se reconcilia con ese Daniel que "no supo estar a la altura" y siente el orgullo de haber superado todos los obstáculos encontrados.

\section{Implicación}

El "yo puedo" de la dimensión anterior no se ve seguido de una actitud proactiva de planificación de los pasos para ejecutarlo.

En la elección de estudios observamos que, en la mayoría de las narraciones, lo prioritario era ir a la Universidad, por encima de cualquier otra opción e independientemente de tener que realizar estudios que no estuvieran relacionados con su interés vocacional. Llegan a la titulación con un gran desconocimiento de la misma.

Ivana muestra su despreocupación por cuestionarse el sentido de estar en sus estudios. Su interés por "estar mejor preparada" parece ser más una aspiración familiar. Ella aspira a mantener su empleo y disfrutar de tiempo libre ("Me matriculé de los 60 créditos, pero después reduje a la mitad porque era mucho trabajo y yo quería tener vida social, quería trabajar").

Candela reconoce que se "mueve mucho por las emociones...saber que voy a estar con gente a gusto" y decide realizar ADE por descarte. Este es el patrón de funcionamiento en la configuración de su trayectoria.

Gisela, accede a Pedagogía sin tener muy claro lo que ésta le puede aportar. Se desmotiva al observar que en la Facultad no responde a su idealización de la educación, lo que le lleva, después del enfado, a buscar alternativas, como la meditación, fuera de la Universidad.

Jano, tiene tomada su decisión desde la infancia: "siempre había querido hacer ADE", y no cabe plantearse la posibilidad de otra opción diferente a la de ir a la Universidad y realizar los mismos estudios que su padre.

Yadira ilustra cómo llega a los estudios universitarios sin saber realmente si es lo que quería hacer: "Hay gente que tiene la suerte de empezar una carrera y que, como ha querido hacer eso toda la vida, le ha entusiasmado....A mí me hubiera gustado de siempre... Pienso: qué suerte tienes".

Ricardo decide estudiar ADE considerando las salidas profesionales de la titulación y sus conocimientos de matemáticas. Configura su trayectoria dejando la responsabilidad a lo que ocurra fuera de sí mismo (aprobar la prueba de acceso, incidencia de la masificación, de la actitud del profesorado...) y con la necesidad del reconocimiento a su esfuerzo.

Servando elige los estudios para descubrir las causas de la situación de desempleo que afecta a su padre ("Mi mayor motivación era porque no entiendo por qué mi padre después de treinta años se ha quedado en paro. ¿Dónde puedo aprender esto? en ADE"). Tampoco se cuestiona la posibilidad de realizar otros estudios diferentes a los universitarios ("... Siempre consideré que la Universidad era donde hay que ir"). Sin embargo, la experiencia no responde a sus expectativas y llega a señalar que no recomienda la formación universitaria tal y como está diseñada y organizada (falta de formación práctica, enseñanza tradicional transmisora...). 
La configuración de los itinerarios formativos se ve marcada en algunos casos por un proceso reflexivo en el que se tienen en cuenta elementos como la "comodidad", la posibilidad de compaginar los estudios con el ocio y las relaciones o el facilitar la consecución de mejores calificaciones (Jano:“...Vi que eran cuatrimestres muy cargados así que decidí descargarlos. Yo no quiero estar tan volcado en la actividad académica porque me apetece hacer inglés, me apetece ir al gimnasio, me apetece quedar con mis amigos"; Mario: "Si quise alargar la carrera un año más fue por comodidad, que para mí estar estudiando es un paso de la vida y que no solo es estudiar"). Montse alude al aprendizaje realizado a partir de su experiencia en el grado: "Me he dividido el máster en dos años, porque después de la experiencia de cuarto veía que me iba a ser imposible o que también lo iba a hacer deprisa y corriendo".

Al final de la trayectoria es cuando comienzan a plantearse cómo puede ser su trabajo. Las referencias que realizan en relación con su proyección de futuro son muy generales. Solo en algunos casos hacen alusión a tipos de puestos de trabajo concretos que les gustaría desempeñar (Jano: “...Quiero un momento de o seguir trabajando, o irme un tiempo fuera. Luego sería hacer un máster, y ya vería de qué"). La falta de implicación de Nubia con su propio proyecto profesional ha impregnado toda la trayectoria, agravándose hacia el final de la titulación ("Y te sientes como aquella niña que no sabía qué hacer cuando metió la primera opción a la selectividad. Y el miedo a fracasar...Y el miedo de equivocarte es terrible"). Para Paloma el problema está en la priorización de intereses, posibilidades, condiciones laborales y de remuneración, confundiéndolas entre sí y se abandona a lo que las circunstancias le puedan traer ("...Me gusta mucho el sector turístico pero está muy mal remunerado, entonces no sé dónde voy a estar... Tengo claro que quiero estar en nivel administrativo y contable, pero no te sé decir dónde quiero trabajar....Sí, pero me veo ahí, me veo en un despacho, con un negocio propio, me veo como jefa de administración en una empresa. Dentro de ese camino, la experiencia y las circunstancias dirán").

Se hace más evidente la presencia de esta dimensión de implicación respecto al futuro en casos (todos de Pedagogía) como el del Biel, quien relaciona su experiencia de voluntariado en el presente con el tipo de trabajo que le gustaría desempeñar en el futuro: "Me gustaría trabajar en un Casal de jóvenes, como yo ahora donde estoy de voluntario... y llevar un proyecto como profesional". Montse aprovecha las informaciones que recibe sobre opciones posteriores a Pedagogía para elegir un máster determinado: "Psicopedagogía me llamaba, pero al estar tan centrado en el colegio.... Me dijeron lo del sociolaboral y vi que tenía salidas y que podía encajar ahi”. Neus reflexiona sobre su trayectoria: "...He reconducido mi vida profesional hacia un fin elaborado, premeditado y suficientemente claro en el que me encuentro predispuesta y totalmente segura de lo que quiero hacer y ser...me gustaría experimentar. Después del máster he de probar más cosas".

\section{Discusión y conclusiones}

Los resultados apuntan a la aportación de la metodología narrativa, a través de la cual los informantes comparten quiénes son y qué les preocupa (Del Corso \& Rehfuss, 2011), para poder comprender de una manera holística, ecológica e histórica el proceso de construcción de la carrera, coincidiendo con las conclusiones a las que han llegado otros investigadores en esta materia (Bimrose \& Hearne, 2012; McMahon et al., 2012; Savickas et al., 2009). Observar y hacer consciente de la forma en la que las personas 
gestionan y negocian sus diferentes transiciones, puede estimular a encontrar el sentido de sus biografías de carrera (Alheit, 2009). La narrativa, en este sentido, puede actuar como un puente que facilita la transición.

Las narraciones han mostrado el carácter evolutivo y multidimensional de la adaptabilidad de la carrera, que se desarrolla a través de la interacción recursiva entre elementos internos, externos y la reflexión. Esta última es la que permite dar sentido a las transiciones y la construcción de la carrera a partir de la experiencia (McMahon et al., 2012; Savickas \& Guichard, 2016). Este carácter multidimensional y sistémico de las transiciones como proceso que lleva a la autorrealización, específicamente en el contexto universitario, es destacado en trabajos como los de González Lorente \& Martínez Clares (2016) o Romero-Rodríguez \& Figuera-Gazo (2016).

También queda patente cómo el estudiantado se adapta a los cambios a través de los recursos y estrategias de adaptabilidad, coincidiendo con los resultados de otras investigaciones (Johnston, 2018; Rudolph et al., 2017). El desarrollo de estos recursos se ve mediado por el contexto (Hamtiaux et al., 2013), en el que la familia tiene un lugar importante (Guan et al., 2016).

Por lo que respecta a la dimensión curiosidad, coincidimos con las escasas investigaciones realizadas respecto a la toma de decisiones de estudiantes universitarios en las que se apunta a que éstas están poco fundamentadas en la exploración. Las decisiones se basan más en una idea muy vaga y general sobre las posibles salidas profesionales que en un proceso de exploración para cuya ejecución muestran serias carencias en hábitos y estrategias de búsqueda de información (Cordón \& García, 2012; Fernández, Peña, Viñuela \& Torío, 2007; Rafuse \& Lanning, 2016).

Las prácticas profesionales son percibidas por los participantes no sólo como un elemento de empleabilidad, sino como un factor que favorece la exploración de sí mismos y dar sentido a la titulación que están realizando, coincidiendo con los resultados obtenidos por Llanes, Figuera \& Torrado (2017).

La debilidad de la exploración lleva a que la planificación de la carrera (dimensión implicación) y la dimensión control presenten también carencias, coincidiendo con los resultados de otras investigaciones como las de Álvarez et al. (2015) y Jiang (2017). Nos encontramos, así, con participantes que están anclados en lo que Rafuse y Lanning (2016) denominan "indecisión constante".

La caracterización como "fracaso" de las experiencias que no responden a sus expectativas refleja la dificultad para aceptar el error (control) como parte de la vida y como oportunidad para el aprendizaje y el crecimiento personal y profesional. En muchos casos explica las situaciones de abandono (Freixa, Llanes \& Venceslao, 2018)

Las narraciones han mostrado que la dimensión confianza ("yo puedo") no siempre se ve acompañada de actuaciones ("yo hago"), coincidiendo con las investigaciones realizadas por Hirschi, Lee, Porfeli y Vondracek (2013) o Merino-Tejedor et al. (2016).

En general, en cuanto a la adaptabilidad de la carrera, podríamos afirmar que los participantes evolucionan de una forma positiva aunque incompleta e insuficiente, para hacer frente a los retos de su carrera. Se mueven principalmente por factores externos que les obligan a posicionarse ante la realidad que se encuentran. Todo el proceso está impregnado de una importante carga emocional. El dominio de las habilidades implicadas en la adaptabilidad de la carrera incide en la implicación y compromiso 
del estudiante durante su trayectoria (Merino-Tejedor et al, 2016), favoreciendo el aprendizaje motivado (Boza \& Méndez, 2013). Las carencias en este proceso dificultan la integración en la vida universitaria tanto a nivel académico como personal (Figuera \& Torrado, 2015a, 2015b). Desde la intervención orientadora se puede acompañar este proceso facilitando los recursos necesarios para que los estudiantes puedan construir su proyecto vital y profesional con sentido (Amado et al., 2017), de una forma consciente y motivada. Este proceso puede incidir, en los primeros años, en el sentimiento de pertenencia a la universidad, al rol de estudiante y a la indagación de la carrera, e ir incorporando elementos de contacto con el mercado de trabajo que permitan a la persona una mejor comprensión de los roles profesionales. La intervención con un enfoque narrativo puede ser de gran valor por su capacidad en la identificación de los temas de vida significativos para el desarrollo de las carreras (Brown \& Bimrose, 2015). Ésta podría ser una línea de investigación a desarrollar en relación con la evaluación del diseño y desarrollo de intervenciones orientadoras realizadas desde este enfoque en el contexto universitario.

Como principales limitaciones de este trabajo debemos señalar la dificultad para poder asegurar la validez interna, así como el número de participantes, si bien teniendo en cuenta las características de la metodología narrativa consideramos que aporta un importante nivel de profundidad en la comprensión de la adaptabilidad de la carrera. Otra limitación es que nos hemos centrado en el ámbito de las Ciencias Sociales y Jurídicas. Futuras investigaciones tendrían que dirigirse a estudiantes de otras áreas científicas, en las que se desarrollan otras culturas universitarias.

\section{Referencias}

Alheit, P. (2009). Biographical learning-Within the new lifelong learning discourse. In K. Illeris (Ed.), Contemporary theories of learning: Learning theorist...in their own words (pp. 116-128). London: Routledge.

Álvarez, P., López, D., \& Pérez-Jorge, D. (2015). El alumnado universitario y la planificación de su proyecto formativo y profesional. Actualidades Investigativas en Educación, 15(1), 1-24. doi: https://doi.org/10.15517/aie.v15i1.16962.

Amado, S., Taveira, M.C., Neves, L., Silva, A.D., Rodrigues, B., \& Costa-Lobo, C. (2017). Efeitos de uma intervençao psicológica vocacional na adaptabilidade de carreira. Revista de Psicologia da Criança e do Adoscente, 8, 93-104.

Barabasch, A., \& Merrill, B. (2014). Cross-cultural Approaches to Biographical Interviews: looking at career transitions and lifelong learning. Research in Comparative and International Education, 9(3), 287-300. doi: https://doi.org/10.2304/ rcie.2014.9.3.287

Bernal, A., \& Cárdenas, A.R. (2009). Influencia de la competencia emocional docente en la formación de procesos motivacionales e identitarios en estudiantes de Educación Secundaria. Una aproximación desde la memoria autobiográfica del alumnado. Revista de Investigación Educativa, 27(1), 203-222.

Bimrose, J., \& Hearne, L. (2012). Resilience and career adaptability: Qualitative studies of adult career counseling. Journal of Vocational Behavior, 81, 338-344. doi: https://doi. org/10.1016/j.jvb.2012.08.002 
Bimrose, J., Watson, M., McMahon, M., Haasler, S., Tomassini, M., \& Suzanne, P. A. (2014). The problem with women? Challenges posed by gender for career guidance practice. International Journal of Educational and Vocational Guidance, 14, 77-88. doi: https://doi.org/10.1007/s10775-013-9256-9.

Boza, A., \& Méndez, J.M. (2013). Aprendizaje motivado en alumnos universitarios: validación y resultados generales de una escala. Revista de Investigación Educativa, 31(2), 331-347. doi10.6018/rie.31.2.163581.

Brown, A., \& Bimrose, J. (2015). Identity Development. En P.J. Hartung, M. Savickas \& W.B. Walsh (Eds.), APA Handbook of career intervention, Vol. 2: Applications. APA Handbooks in Psychology (pp.242-254). Washington D.C. US: APA.

Brown A., Bimrose, J., Barnes, S.A., \& Hughes, D. (2012). The role of career adaptabilities for mid-career changers. Journal of Vocational Behavior, 80, 754-761. doi: https:// doi.org/10.1016/j.jvb.2012.01.003.

Cordón, A.R., \& García, F. (2012). Evidencias empíricas en el proceso de elección de los estudios universitarios. Enseñanza E Teaching, 30(2), 45-62.

Del Corso, J., \& Rehfuss, M.C. (2011). The role of narrative in career construction theory. Journal of Vocational Behavior, 79, 334-339. doi: https://doi.org/10.1016/j. jvb.2011.04.003.

Douglass, R.P., \& Duffy, R.D. (2014). Calling and career adaptability among undergraduate students. Journal of Vocational Behavior, 86, 58-65. doi: https://doi.org/10.1016/j. jvb.2014.11.003.

Figuera, P., \& Torrado, M. (2015a). Análisis longitudinal del proceso de transición a la Universidad. Estudio de un caso. En P. Figuera (Coord.), Persistir con éxito en la Universidad (pp. 97-112). Barcelona: Laertes.

Figuera, P., \& Torrado, M. (2015b). The Transition to University of At-Risk Groups in Spain: The Case of Students from Vocational Education and Training. Revista de cercetare [i interven] ie social, 49, 23-40.

Fernández, C.M., Peña, J.V., Viñuela, M.P., \& Torío, S. (2007). Los procesos de orientación escolar y la toma de decisiones académica y profesional. Revista Complutense de Educación, 18(2), 87-103.

Freixa, M., \& Dorio, I. (2015). ¿Qué cuentan los estudiantes sobre su experiencia universitaria? En T. Raitz y P. Figuera (Orgs.), Transições dos estudantes: reflexões iberoamericanas (pp. 147-158). Curitiba, Brasil: CRV.

Freixa, M., Llanes, J., \& Venceslao, M. (2018). El abandono en el recorrido formativo del estudiante de ADE de la Universidad de Barcelona. Revista de Investigación Educativa, 36(1), 185-202. doi: https://doi.org/ 10.6018/rie.36.1.278971.

Glaser, B. G., \& Strauss, A. (2009). The discovery of grounded theory: Strategies for qualitative research. New Jersey: Transaction Publishers.

González Lorente, C., \& Martínez Clares, P. (2016). Expectativas de futuro laboral del universitario de hoy: un estudio internacional. Revista de Investigación Educativa, 34(1), 167-183. doi: https://doi.org/10.6018/rie.34.1.232071.

Guan, P., Capezio, A., Restubog, S.L.D., Read, S., Lajomb, J.A.L., \&, Li, M. (2016). The role of traditionality in the relationships among parental support, career decisionmaking self-efficacy and career adaptability. Journal of Vocational Behavior, 94, 114-123. doi: https://doi.org/10.1016/j.jvb.2016.02.018. 
Hamtiatux, A., Houssemand, C., \& Vrignaud, P. (2013). Individual and career adaptability: Comparing models and measures. Journal of Vocational Behavior, 83, 130-141. doi: https://doi.org/10.1016/j.jvb.2013.03.006.

Hirschi, A., Lee, B., Porfeli, E.J., \& Vondracek, F.W. (2013). Proactive motivation and engagement in career behaviors: Investigating direct, mediated, and moderated effects. Journal of Vocational Behavior, 83, 31-40. doi: https://doi.org/10.1016/j. jvb.2013.02.003 .

Ibarra, H., \& Barbulescu, R. (2010). Identity As Narrative: Prevalence, Effectiveness, and Consequences of Narrative Identity Work in Macro Work Role Transitions. Academic Management Review, 35(1), 135-154.

Jian, Z. (2017). Proactive personality and career adaptability: The role of thriving at work. Journal of Vocational Behavior, 98, 85-97. doi: https://doi.org/10.1016/j.jvb.2016.10.003.

Johnston, C.S. (2018). A systematic review of the literature and future outlook. Journal of Career Assessment, 26(1), 3-30. doi: https://doi.org/10.1177/1069072716679921.

Llanes, J., Figuera, P., \& Torrado, M. (2017). Desarrollo de la empleabilidad y gestión personal de la carrera de graduados en Pedagogía. Revista Española de Orientación y Psicopedagogía, 28(2), 46-60. doi: https://doi.org/10.5944/reop.vol.28.num.2.2017.20118

Martínez-Clares, P., Pérez-Cusó, F.J., \& Martínez-Juárez, M. (2014). Orientación Profesional en Educación Secundaria. Revista Electrónica Interuniversitaria de Formación del Profesorado, 17(1), 57-71. doi: https://doi.org/10.6018/reifop.17.1.198841

McAdams, D.P., \& Guo, J. (2014). How Shall I Live? Constructing a life story in the College years. New Directions for Higher Education, 166, 15-23.

McMahon, M., Watson, M., \& Bimrose, J. (2012). Career adaptability: A qualitative understanding from the stories of older women. Journal of Vocational Behavior, 80, 762-768. doi: https://doi.org/10.1016/j.jvb.2012.01.016

Merino-Tejedor, E., Hontangas, P.M., \& Boada-Grau, J. (2016). Career adaptability and its relation to self-regulation, career construction, and academic engagement among Spanish university students. Journal of Vocational Behavior, 93, 92-102. doi: https:// doi.org/10.1016/j.jvb.2016.06.001

Merino-Tejedor, E., Hontangas, P.M., \& Petrides, K.V. (2017). La adaptabilidad a la carrera media el efecto de la inteligencia emocional sobre el compromiso académico. Revista Psicodidáctica, 23(2), 77-85. doi: https://doi.org/10.1016/j.psicod.2017.10.001

Monteiro, S., \& Da Silva, L.S. (2015). The relation of career adaptability to work experience, extracurricular activities, and work transition in Portuguese graduate students. Journal of Vocational Behavior, 91, 106-112. doi: https://doi.org/10.1016/j. jvb.2015.09.006

Nilforooshan, P., \& Salimi, S. (2016). Career adaptability as a mediator between personality and career engagement. Journal of Vocational Behavior, 94, 1-10. doi: https:// doi.org/10.1016/j.jvb.2016.02.010

Nota, L., \& Rossier, J. (2015). Handbook of Life Design: From Practice to Theory and from Theory to Practice. Göttingen: Hogrefe. doi: https://doi.org/10.11588/nicenl.2015.1.20479.

Perera, H.N., \& Mcllveen, P. (2017). Profiles of career adaptativity and their relations with adaptability, adapting and adaptation. Journal of Vocational Behavior, 98, 70-84. doi: https://doi.org/10.1016/j.jvb.2016.10.001 
Presbitero, A., \& Quita, C. (2017). Expatriate career intentions: Links to career adaptability and cultural intelligence. Journal of Vocational Behavior, 98, 118-126. doi: https:// doi.org/10.1016/j.jvb.2016.11.001.

Rafuse, A., \& Lanning, R. (2016). Knowledge and risk: choices and decisions in the career selection process among University students. The Canadian Journal of Career Devolpment, 15(2), 32-42.

Romero-Rodríguez, S., \& Figuera-Gazo, P. (2016). Orientación en la Universidad. En A. Manzanares-Moya \& C. Sanz-López (Dir.), Orientación Profesional. Fundamentos y estrategias (pp. 163-181). Madrid: Walters Kluwer.

Romero-Rodríguez, S., Figuera-Gazo, P., \& Freixa-Niella, M. (2017). Narrating emerging adulthood at University. En B. Merrill, J. González-Monteagudo, A. Nizinska, A. Galimberti \& M.A. Ballesteros-Regaña (Eds.), Adult learning, Educational careers and Social change, (pp. 26-40). Seville: University of Seville/ESREA.

Rudolph, C.W., Lavigne, K.N., Katz, I.M., \& Zacher, H. (2017). Linking dimensions of career adaptability to adaptation results: A meta-analysis. Journal of Vocational Behavior, 102, 151-173. doi: https://doi.org/10.1016/j.jvb.2017.06.003.

Rudolph, C.W., Lavigne, K.N., \& Zacher, H. (2017). Career adaptability: A meta-analysis of relationships with measures of adaptivity, adapting responses, and adaptation results. Journal of Vocational Behavior, 98, 17-34. doi: https://doi.org/10.1016/j. jvb.2016.09.002.

Sandín, M.P. (2000). Criterios de validez en la investigación cualitativa: de la objetividad a la solidaridad. Revista de Investigación Educativa, 18(1), 223-242.

Sandín, M.P. (2010). Investigación cualitativa en educación: Fundamentos y tradiciones. Madrid: McGraw-Hill.

Santos, A.V., \& Cardenal, M.E. (2012). Los Sujetos, la Educación Superior y los Procesos de Transición. Aportaciones del enfoque biográfico. Revista Lusófona de Educação, 21, 121-138.

Savickas, M.L., Nota, L., Rossier, J., Dauwalder, J.P., Duarte, M.E., Guichard, J....van Vianem, A.E.M. (2009). Life designing: A paradigm for career construction in the 21st century. Journal of Vocational Behavior, 75, 239-250. doi: https://doi.org/10.1016/j. jvb.2009.04.004.

Savickas, M.L. \& Guichard, J. (2016). Symposium introduction: reflexivity in life designing interventions. Journal of Vocational Behavior, 97, 1-2. doi: https://doi.org/10.1016/j. jvb.2016.07.015.

Savickas, M.L., \& Porfeli, E.J. (2012). Career Adapt-Abilities Scale: Construction, reliability, and measurement equivalence across 13 countries. Journal of Vocational Behavior, 80, 661-673. doi: https://doi.org/10.1016/j.jvb.2012.01.011

Shulman, S., Vasalampi, K., Barr, Z., Livne, Y., Nurmi, J.E., \& Pratt, M.W. (2014). Typologies and precursors of career adaptability patterns among emerging adults: A seven-year longitudinal study. Journal of Adolescence, 37, 1505-1515. doi: https:// doi.org/10.1016/j.adolescence.2014.06.003.

Šverko, I., \& Babarović, T. (2016). Integrating personality and career adaptability into vocational interest space. Journal of Vocational Behaviour, 94, 89-103. doi: https://doi. org/10.1016/j.jvb.2016.02.017 
Whiston, S.C., Feldwisch, R.P., Evans, K.M., Blackman, C.S., \& Gilman, L. (2015). Older professional women`s views on work: A qualitative analysis. The Career Development Quarterly, 63, 98-112. doi: https://doi.org/ 101002/cdq.12007.

Zacher, H. (2016). Within-person relationships between daily individual and job characteristics and daily manifestations of career adaptability. Journal of Vocational Bechavior, 92, 105-115. doi: https://doi.org/10.1016/j.jvb.2015.11.013.

Yin, R.K., (1984). Case Study Research: Design and Methods. Beverly Hills, Calif: Sage Publications.

Fecha de recepción: 22 de febrero de 2018.

Fecha de revisión: 5 de marzo de 2018.

Fecha de aceptación: 7 de mayo de 2018. 